Corrigendum

\title{
Corrigendum to "Clinical and Preclinical Cognitive Function Improvement after Oral Treatment of a Botanical Composition Composed of Extracts from Scutellaria baicalensis and Acacia catechu"
}

\author{
Mesfin Yimam, ${ }^{1}$ Bruce P. Burnett, ${ }^{2}$ Lidia Brownell, ${ }^{1}$ and Qi Jia ${ }^{1}$ \\ ${ }^{1}$ Unigen, Inc., 3005 1st Ave., Seattle, WA 98121, USA \\ ${ }^{2}$ Entera Health, Inc., 2000 Regency Parkway, Ste. 200, Cary, NC 27518, USA \\ Correspondence should be addressed to Mesfin Yimam; myimam@unigen.net
}

Received 6 April 2017; Accepted 26 April 2017; Published 3 July 2017

Copyright (C) 2017 Mesfin Yimam et al. This is an open access article distributed under the Creative Commons Attribution License, which permits unrestricted use, distribution, and reproduction in any medium, provided the original work is properly cited.

In the article titled "Clinical and Preclinical Cognitive Function Improvement after Oral Treatment of a Botanical Composition Composed of Extracts from Scutellaria baicalensis and Acacia catechu" [1], there is an error in a sentence in the abstract section. The sentence, "In a separate human clinical trial, test subjects orally given $300 \mathrm{mg}$ of UP326 BID for 30 days showed marked improvement in speed and accuracy of processing complex information in computer tasks and reduced their standard deviation of performance compared to baseline and the placebo group" should be corrected to "In a separate human clinical trial, test subjects orally given $300 \mathrm{mg}$ of UP326 per day for 30 days showed marked improvement in speed and accuracy of processing complex information in computer tasks and reduced their standard deviation of performance compared to baseline and the placebo group."

\section{References}

[1] M. Yimam, B. P. Burnett, L. Brownell, and Q. Jia, "Clinical and preclinical cognitive function improvement after oral treatment of a botanical composition composed of extracts from Scutellaria baicalensis and Acacia catechu," Behavioural Neurology, vol. 2016, Article ID 7240802, 9 pages, 2016. 


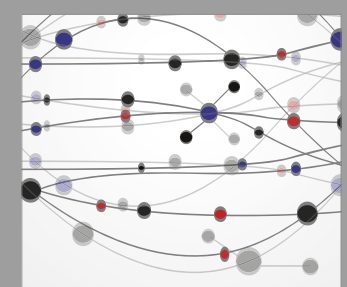

The Scientific World Journal
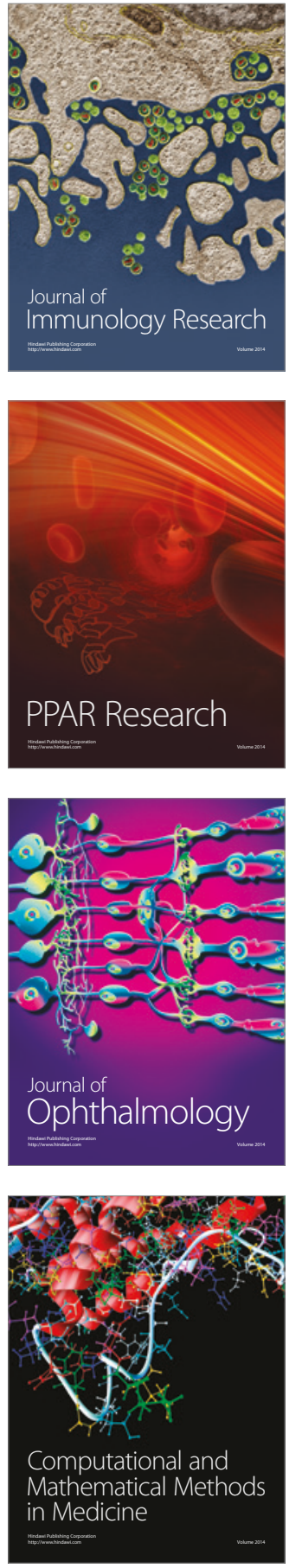

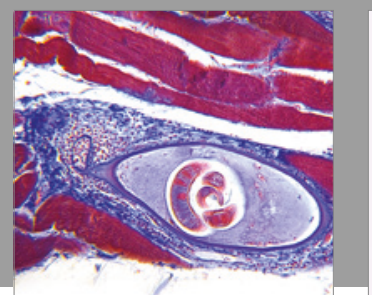

Gastroenterology Research and Practice
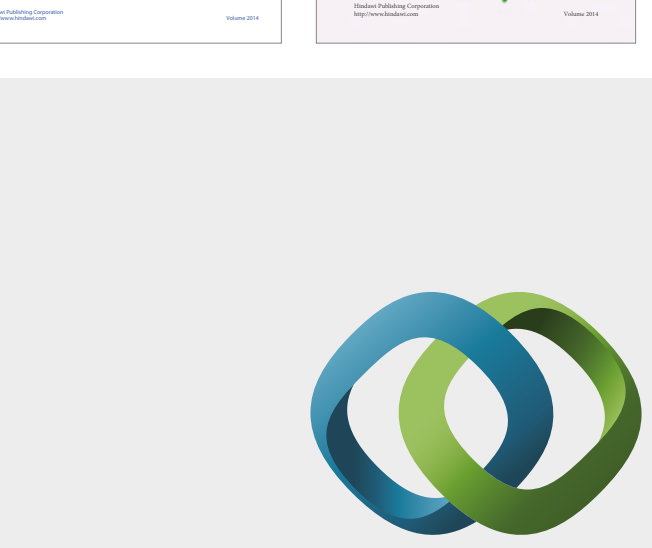

\section{Hindawi}

Submit your manuscripts at

https://www.hindawi.com
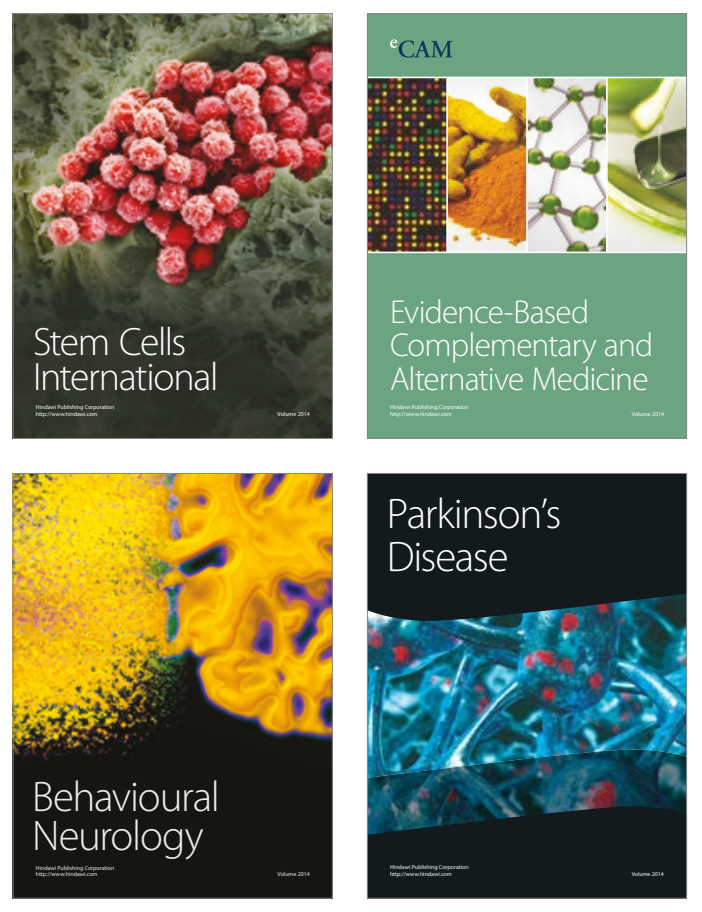
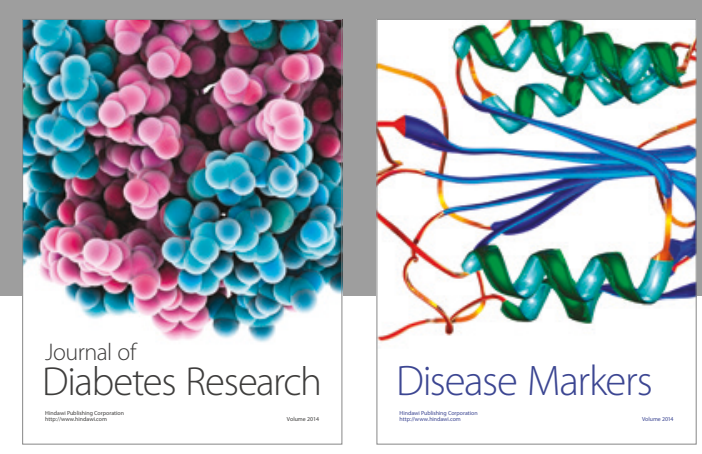

Disease Markers
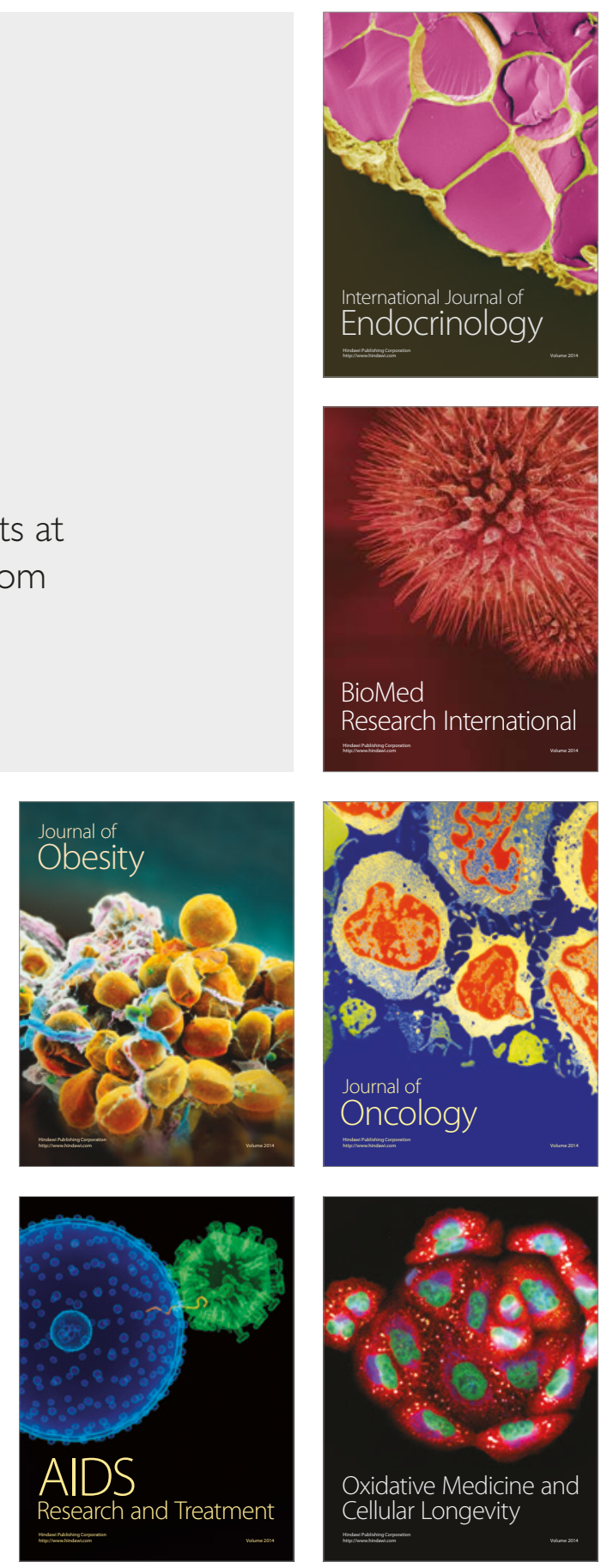